\title{
Integrating lattice and gap plasmonic modes to construct dual-mode metasurfaces for enhancing light-matter interaction
}

\author{
Limin $\operatorname{Lin}^{1 \dagger}$, Jiancai Xue ${ }^{1 \dagger}$, Haofei $\mathrm{Xu}^{1}$, Qian Zhao ${ }^{1}$, Wenbo Zhang ${ }^{1}$, Yaqin Zheng ${ }^{1}$, Lin $\mathrm{Wu}^{2}$ and \\ Zhang-Kai Zhou ${ }^{1 *}$
}

\begin{abstract}
Photonic structures with optical resonances beyond a single controllable mode are strongly desired for enhancing light-matter interactions and bringing about advanced photonic devices. However, the realization of effective multimodal photonic structures has been restricted by the limited tunable range of mode manipulation, the spatial dispersions of electric fields or the polarization-dependent excitations. To overcome these limitations, we create a dualmode metasurface by integrating the plasmonic surface lattice resonance and the gap plasmonic modes; this metasurface offers a widely tunable spectral range, good overlap in the spatial distribution of electric fields, and polarization independence of excitation light. To show that such dual-mode metasurfaces are versatile platforms for enhancing lightmatter interactions, we experimentally demonstrate a significant enhancement of second-harmonic generation using our design, with a conversion efficiency of 1-3 orders of magnitude larger than those previously obtained in plasmonic systems. These results may inspire new designs for functional multimodal photonic structures.
\end{abstract}

Keywords: dual-mode metasurfaces, gap plasmon, surface lattice resonance, independently dual-mode manipulation, enhancement of light-matter interaction

\section{INTRODUCTION}

Because of their intrinsic ability to confine electric fields (EFs) at a subwavelength scale, the resonant modes of photonic structures can sustain large EF enhancements and have brought about fruitful achievements in both fundamental and applied optics [1-7]. A variety of novel optical phenomena-such as Fano resonance [8], plasmon-exciton strong coupling [9], invisibility cloaking [10], giant circular dichroism [11], and large nonlinear response [12] - have been demonstrated using appropriately tailored resonant modes. These investigations have led to a diversity of unprecedented applications, including metalenses [13], single-photon sources [14], integrated quantum circuits [15], nanolasers [16], and biochemical-sensing installations [17], and thus bridge the gap between fundamental optics and the real-world applications.

In the majority of previous studies, a single resonant mode is utilized. An important goal in photonics is to improve the resonances of photonic structures from a single-resonant mode to on-demand dual modes. To date, great efforts have been devoted to realizing this goal, but curial problems still remain. The dual-resonant modes of most such structures cannot be modulated independently, and in many dual-mode cases-such as the "templar cross" antenna [18] or the three rectangular arms [19]there is actually only one spectrally tunable mode. These drawbacks in mode modulation severely limit the design of an on-demand dual-mode photonic structure with targeted spectral bands. Furthermore, there have always been limitations on the excitations of these dual modes, such as the varied incident polarizations required for each mode or the spatial mismatch of the EF enhancement distributions; this inevitably hinders the ability of the two modes to function simultaneously.

To address the abovementioned problems, we propose constructing a dual-mode metasurface (DMM) by in-

\footnotetext{
${ }^{1}$ State Key Laboratory of Optoelectronic Materials and Technologies, School of Physics, Sun Yat-sen University, Guangzhou 510275, China

${ }^{2}$ Institute of High Performance Computing, A*STAR (Agency for Science, Technology and Research), 1 Fusionopolis Way, Connexis, Singapore 138632, Singapore

† These authors contributed equally to this work.

*Corresponding author (email: zhouzhk@mail.sysu.edu.cn)
} 
tegrating the lattice and gap plasmonic modes. Gap plasmons distinguish themselves by offering remarkable enhancement of the EF in the structural gap [20-23], whereas the plasmonic surface lattice resonance possesses unique advantages for achieving high-quality factors and lowering optical losses [24-26]. Additionally, these two modes can both be designed to be optically polarizationindependent in the DMM. It is worth mentioning that, since plasmonic surface lattice modes always stem from the coupling of a localized plasmonic resonance, in previous studies, such lattice modes only appear at spectral positions around a localized mode. However, under our design, with the DMM consisting of a two-dimensional array of sandwich heterostructures ( $\mathrm{Ag}$ disks $/ \mathrm{SiO}_{2} / \mathrm{Ag}$ film), the two resonances can be independently tunable from the visible to the near-infrared region by adjusting the structural parameters. Another advantage of our DMM is that the enhanced EF distributions of the two modes overlap well in space. The combination of these features makes the DMM a promising versatile platform for light-matter interactions. To demonstrate the enhancement of the light-matter interaction, the two modes of the DMM are tuned to simultaneously enhance second-harmonic generation (SHG), with an enhancement factor as high as $5 \times 10^{3}$, obtaining a new record of SHG conversion efficiency in a plasmonic system (1-3 orders of magnitude larger than those in previous reports). Moreover, it is found that modal strong coupling can be achieved by tuning the two modes to an overlapped spectral region, giving rise to a great potential for studying the coherent manipulation of the strong coupling. Our work not only develops a promising platform for enhancing light-matter interactions but also offers new inspirations for the design of multimodal photonic structures.

\section{EXPERIMENTAL SECTION}

\section{Numerical simulation}

The simulation results were calculated using the finitedifference time-domain (FDTD) method [27] via a commercially available FDTD simulation software package from Numerical Solutions. The permittivity of silver was taken from Palik [28], and the refractive index of the silica was measured as 1.45 .

\section{Optical and morphology characterizations}

The experimental reflection and SHG spectra were measured via a spectrograph (SP2500, Princeton Instruments) connected to a microscope (Olympus BX51M, Olympus
Inc.). The objective of $5(\mathrm{NA}=0.15)$ was adopted in the measurements for the reflection spectra, whereas an objective of $50(\mathrm{NA}=0.8)$ was used for the SHG spectra. The scanning electron microscopy (SEM) images were taken by a Zeiss Auriga-39-34 (Oberkchen, Germany) microscope operating at $5.0 \mathrm{kV}$.

\section{Fabrication}

First, a 200-nm-thick silver reflecting layer was deposited onto a silicon substrate by an electron beam evaporation system (DE400, DE Technology) with a speed of $2 \AA \mathrm{s}^{-1}$. A 24-nm-thick $\mathrm{SiO}_{2}$ film was deposited onto the silver reflector using inductive coupled plasma enhanced chemical vapor deposition (ICP-PECVD) (PlasmaPro System 100 , Oxford) under $80^{\circ} \mathrm{C}$. The flow rates of $\mathrm{N}_{2} \mathrm{O}$ and $\mathrm{SiH}_{4}$ are 13.0 and 4.0 s.c.c.m., respectively. Second, an electron resist (ZEP) layer was spin-coated onto the $\mathrm{SiO}_{2}$ film, followed by a baking process at $170^{\circ} \mathrm{C}$ for $3.5 \mathrm{~min}$. The designed patterns of the nanodisk arrays were written in the ZEP resist with a current of $2 \mathrm{nA}$, a dose of $300 \mathrm{C} \mathrm{cm}^{-2}$, a resolution of $1 \mathrm{~nm}$, and a beam-step size of $5 \mathrm{~nm}$ using an electron beam lithography (EBL) system (EBPG5000+, Riath). The sample was subsequently developed in dimethyl benzene for $70 \mathrm{~s}$ and then immersed in isopropyl alcohol for $30 \mathrm{~s}$ to form the designed distribution of unit holes in the ZEP resist. Third, 20-nmthick silver was deposited into the holes in the ZEP resist by an electron beam evaporation system (DE400, DE Technology) with a speed of $2 \AA \mathrm{s}^{-1}$. Finally, the sample was soaked in an acetone-with-water bath at $45^{\circ} \mathrm{C}$ for approximately $15 \mathrm{~min}$ to peel off the ZEP resist, leaving behind the nanodisks.

\section{RESULTS AND DISCUSSION}

\section{Mechanism and overall features of the dual-mode metasurfaces}

To simultaneously sustain the lattice and gap plasmonic modes, the DMMs were constructed by Ag nanodisks arranged in a tetragonal array on an Ag film substrate of $200 \mathrm{~nm}$ in thickness with a spacer layer of $\mathrm{SiO}_{2}$ (Fig. 1a). Four key parameters were applied to control these two modes, namely, the thickness of the $\mathrm{SiO}_{2}$ layer $(g)$, the height $(H)$ and diameter $(D)$ of the unit Ag nanodisks, and the period of nanodisk array $(P)$. Generally, it is found that the smaller the values of $g$ and $H$, the larger the EF enhancements (Fig. S1). Thus, we set $g$ and $H$ to 24 and $20 \mathrm{~nm}$, respectively, approaching the limit of our fabrication technology while satisfying reproducibility. Thereafter, the resonant modes in our DMMs were 
a
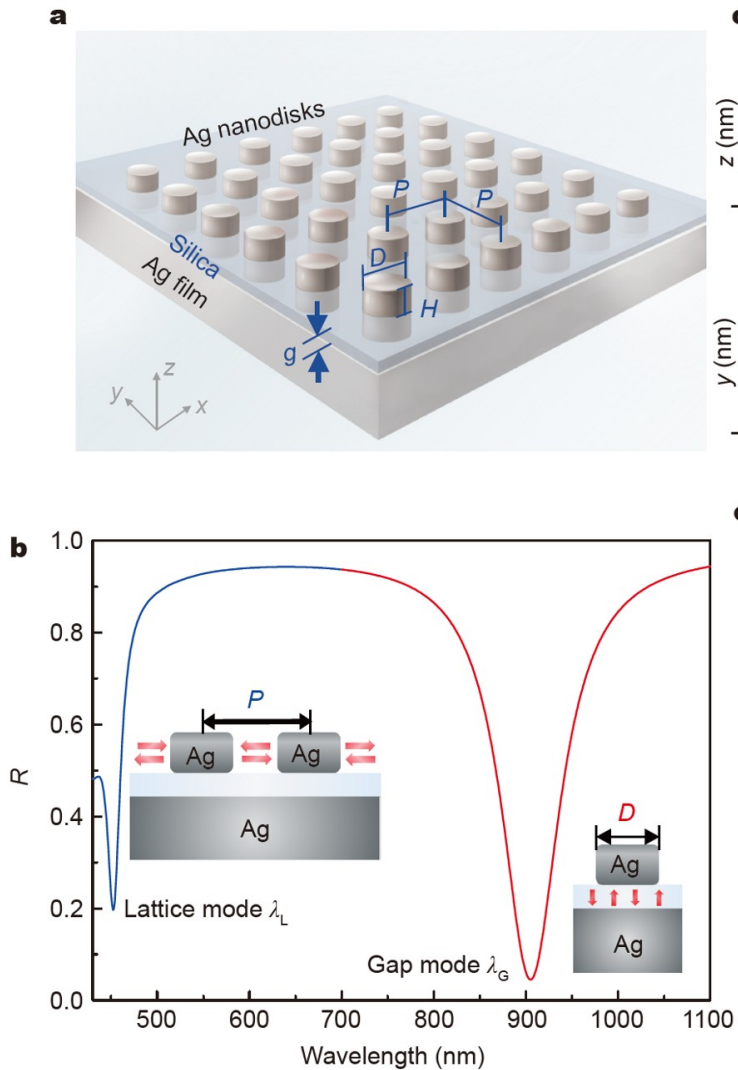

c
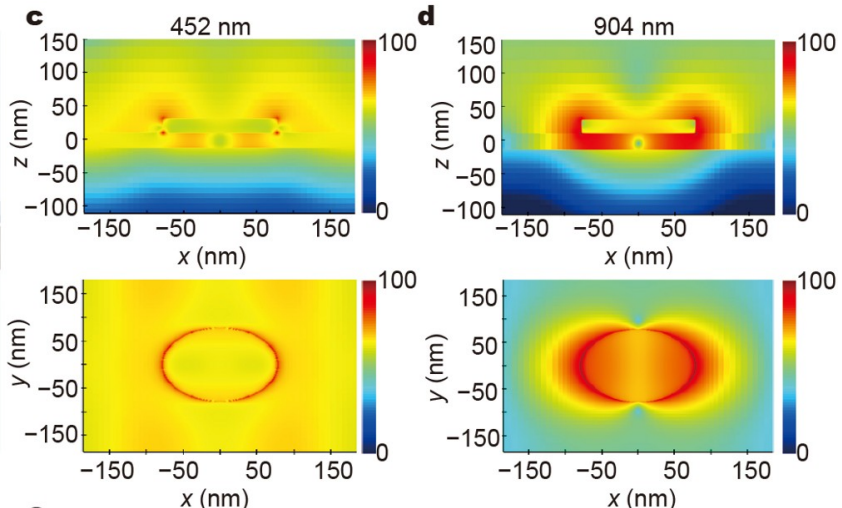

e

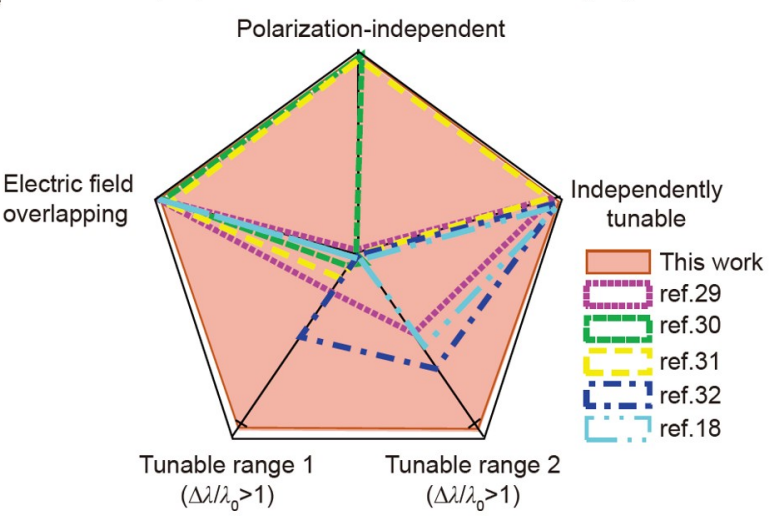

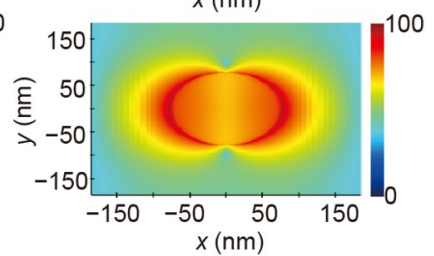

Figure 1 Dual-mode metasurfaces combining lattice and gap plasmonic modes. (a) A schematic of our DMM structure. (b) One typical simulationreflection spectrum of the DMMs. The lattice mode (solid blue curve) is tunable by changing the array period $(P)$, whereas the gap mode (solid red curve) is tunable by varying the diameter $(D)$ of nanodisks. The local electric field enhancement of the lattice (c) and the gap (d) plasmonic modes in the $x-z$ and $x-y$ planes, plotted in log10 scale. (e) Key features of our DMM and comparisons with previous dual-mode photonic structures. The purple dotted, green short-dashed, yellow dashed, blue dashed-dotted, and cyan dashed-dotted lines represent data taken from Refs [29-32] and [18]. The orange solid line and area correspond to the results in this work.

controlled by varying the values of $P$ and $D$.

To identify the origins of the dual-resonant modes in the DMMs, typical values for $P$ and $D$ were selected (i.e., $P=350 \mathrm{~nm}$ and $D=160 \mathrm{~nm}$ ) for theoretical analysis, and the corresponding reflection spectrum (Fig. 1b) exhibits two distinct valleys at 452 and $904 \mathrm{~nm}$ (i.e., in the blueand infrared-light regions). We can thus conclude that these two reflection valleys respectively originate from the lattice and gap plasmonic modes. This conclusion is based on two main factors. First, the resonant mode in the blue region does not appear in an individual structure of the $\mathrm{Ag}$ disk/ $\mathrm{SiO}_{2} / \mathrm{Ag}$ film, but the infrared still exists and can be tuned by the $D$ of the single nanodisk (Figs S2 and S3). This fact indicates that the blue resonance mode is the lattice mode caused by the periodic features of the plasmonic array [24]. Second, for the array of the Ag disk/ $\mathrm{SiO}_{2} / \mathrm{Ag}$ film, the resonant mode in the infrared region gradually disappears with the increase of $\mathrm{SiO}_{2}$ thickness
(Fig. S4), agreeing well with the optical behavior of the gap plasmon resonance [21]. Therefore, unlike most previous studies of dual-mode nanostructures, we successfully demonstrate a combination of the lattice and gap plasmonic modes on one metasurface, with resonant wavelengths defined as $\lambda_{\mathrm{L}}$ and $\lambda_{\mathrm{G}}$, respectively.

By integrating the lattice and gap modes, the DMMs demonstrate three valuable features manifested in the following. First, both of the lattice and gap modes have large EF enhancements, and their EF enhancement distributions overlap spatially around the edge of the nanodisk, allowing the possibility for these two modes to work simultaneously (Fig. 1c, d). Note that the EF enhancement is $E / E_{0}$, where $E_{0}$ is the intensity of the incident $\mathrm{EF}$ in a vacuum environment and $E$ is that of an $\mathrm{EF}$ in the presence of nanostructures. Second, since the lattice and gap modes can be independently manipulated by separately adjusting different structural parameters, the 
DMMs enable an unprecedentedly large range of spectral tuning for both modes. Third, because of the high symmetry of our metasurface unit (i.e., the nanodisk structure), the optical responses of the lattice and gap modes in our DMMs are polarization-independent, making it function well under incident light with arbitrary polarizations. Fig. 1e summarizes these features, and more detailed discussions are performed in the following text, as well as in Table S1 in the Supplementary information.

\section{On-demand mode manipulation of the dual-mode metasurfaces}

From the perspective of applications, photonic structures with large spectral tunability and on-demand generation of resonant modes are highly desired; these requirements can be well satisfied by our DMMs because of their advantages for mode manipulation. As clearly shown in Fig. 2a, b, the lattice and gap modes mainly depend upon $P$ and $D$, respectively. To be specific, when $D$ is fixed at $160 \mathrm{~nm}, \lambda_{\mathrm{L}}$ can vary from 403 to $640 \mathrm{~nm}$ with $P$ changing from 260 to $600 \mathrm{~nm}$; however, the gap mode is stable with varied $P$ (Fig. 2a). Similarly, if $P$ is a pre-set constant, the gap mode (or $\lambda_{\mathrm{G}}$ ) distinctly red shifts with increasing $D$, whereas the lattice mode is only slightly perturbed (Fig. 2b). These facts indicate that it is possible to control the lattice and gap modes by sequentially varying the values of $P$ and $D$, respectively.

The different relationships between the gap and lattice
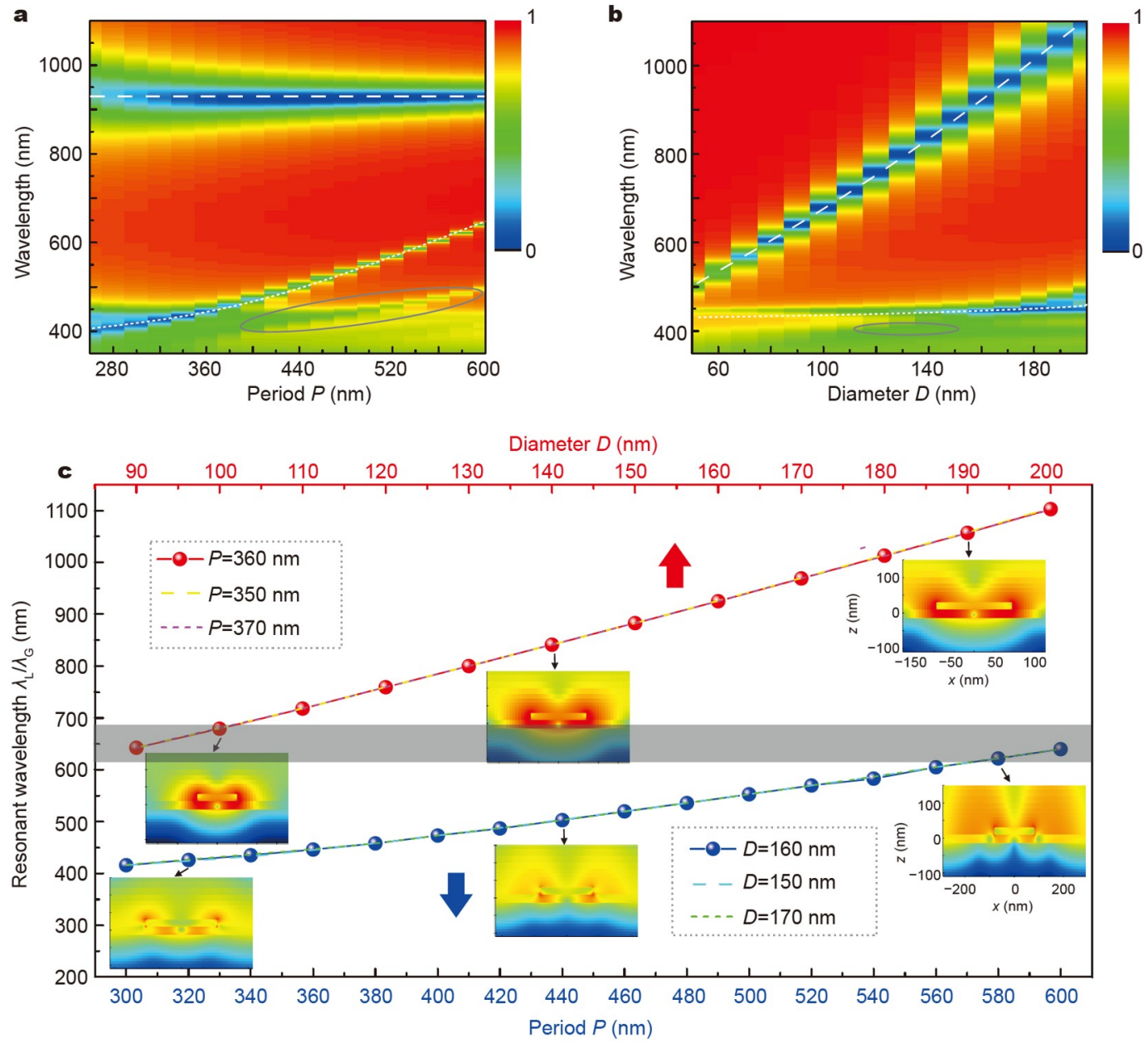

Figure 2 Mode manipulation in our dual-mode metasurfaces. (a) Contour plots of the reflection spectra calculated using FDTD when $D$ is fixed at $160 \mathrm{~nm}$ and $P$ changes from 260 to $600 \mathrm{~nm}$. (b) Contour plots of the reflection spectra calculated using FDTD when $P$ is fixed at $360 \mathrm{~nm}$ and $D$ changes from 50 to $200 \mathrm{~nm}$. White lines are the guides for identifying different modes, including the lattice mode (white dotted line) and the gap mode (white dashed line). The localized plasmon resonance mode of the Ag nanodisk is marked by the dashed ellipse. (c) The $\lambda_{\mathrm{L}}-P$ (blue) and $\lambda_{\mathrm{G}}-D$ (red) relationships. Data collected from (a) and (b) are plotted as lines of blue and red symbols. The gray rectangle shows the spectral overlap of $\lambda_{\mathrm{L}}$ and $\lambda_{\mathrm{G}}$. Insets: $\mathrm{EF}$ diagrams with the same scale bar showing that $\mathrm{EF}$ enhancement hardly changes with variations of $D$ and $P$. 
modes and the structural parameters $(D$ and $P)$ are determined by their origins. Meanwhile, the gap mode stems from the single-particle-on-mirror structure and is mainly determined by the parameters of the nanodisk and the gap; hence, $\lambda_{\mathrm{G}}$ is easily tuned by changing $D$ but not $P$. The lattice mode, meanwhile, results from the far-field diffractive coupling of the scattered field, with the resonance arising when the EF around a cell particle is in phase with the scattering fields from its nearest neighboring particles, meaning that the lattice mode is inherently related to the period of the plasmonic array. This origin determines that $\lambda_{\mathrm{L}}$ follows the changes of $P$ and is not sensitive to $D$.

To more vividly demonstrate the relationship between the lattice mode (and gap mode) and $P$ (and $D$ ), Fig. 2c displays the $\lambda_{\mathrm{L}}-P$ and $\lambda_{\mathrm{G}}-D$ relationships. For the three $\lambda_{\mathrm{L}}$ $-P$ curves, their $D$ values are set as 150,160 , and $170 \mathrm{~nm}$. Surprisingly, these three curves are nearly the same, and similar observations can also be obtained from the three $\lambda_{\mathrm{G}}-P$ curves with $P$ values of 350,360 , and $370 \mathrm{~nm}$. Since current nanofabrication techniques can easily yield a precision within $\pm 5 \mathrm{~nm}$ [5], the theoretical results in Fig. $2 \mathrm{c}$ indicate that the DMMs have a good tolerance for fabrication error, greatly benefiting their device applications. Moreover, as shown by the insets of EF distribution in Fig. 2c, the EF enhancement performance is barely affected by structure parameter variation, ensuring the applicability of the DMMs in a broad spectral band.

To characterize the tunability of the resonant modes in a more systematic way, we defined a relative spectral range factor, $\Delta \lambda / \lambda_{0}$, where $\Delta \lambda=\lambda_{\max }-\lambda_{0}$ (with $\lambda_{\max }$ and $\lambda_{0}$ being the maximum and minimum resonant wavelengths, respectively). We find that the values of $\Delta \lambda / \lambda_{0}$ for both lattice and gap modes can be over 1 in our DMM systems (Fig. S5a), demonstrating extremely wide tunable ranges. In fact, the tunable range of the modes can be further improved, and the resonant wavelengths of the dual modes can be expanded to the infrared region if proper structure optimization-such as changing Ag to other metals-is applied (Fig. S5b). It should be noted that a localized plasmon resonance of the Ag nanodisk in the blue region can be observed (marked by the dashed ellipse in Fig. 2a, b), but it is not used to construct the DMMs because of its much smaller tunable range than that of the lattice mode. Further discussion can be found in Fig. S6.

\section{Polarization-independent property of the dual-mode metasurfaces}

We now turn to optical polarization independence in the
DMM system. To increase the general applicability of photonic structures, realizing the polarization-independent excitation of their photonic modes is of great importance. This property can not only simplify application requirements but also boost the robustness of photonic devices [33-35]. Herein, we delicately apply metasurfaces with high symmetry to construct the DMMs, enabling both the lattice and gap modes to be excited in arbitrary polarizations. As demonstrated by Fig. 3a (which shows the reflection spectra of DMMs under incident light with linear polarizations of $0^{\circ}, 45^{\circ}$, and $90^{\circ}$ ), the two DMM modes exhibit no difference under polarization variation (data regarding other polarization angles can be found in Fig. S7a). Such polarization independence stems from the structural symmetry of the DMMs, which can be further understood from the electric field distributions of polarization angles (the insets of Fig. 3a and Fig. S8). If such structural symmetry is broken (for instance, when the nanodisks are replaced by nanorods), the polarization-related optical response will be correspondingly changed (Fig. $3 \mathrm{~b}$ and Fig. S9). Experimentally, the polarization independence feature can be easily validated by the involvement of the polarizer, as shown in Fig. $3 \mathrm{c}$ and Fig. S7b. Polarization independence is quite valuable for real-world applications because of the diminished restriction to the applied light source.

\section{Giant optical enhancement enabled by dual-mode resonances}

In most cases of enhancing an optical process, giant enhancements require EF enhancements to be generated at different wavelengths simultaneously-for example, at the wavelengths of both pumping and radiation for nonlinear processes or photoluminescence enhancement [36-38]. Such requirements can be perfectly met by our DMMs because of their superiority, as mentioned above. As proof of the DMM's optical enhancement performance, we characterized its SHG enhancement. The SHG can be regarded as a coherent three-wave mixing process in which two photons of fundamental wavelength $\left(\lambda_{\mathrm{FW}}\right)$, assisted by a plasmonic resonance, are absorbed and induce a radiative decay assisted by another plasmonic resonance at the second-harmonic wavelength $\left(\lambda_{\mathrm{SHG}}=\lambda_{\mathrm{FW}} /\right.$ 2 ) with the generation of a second-harmonic photon. Therefore, to achieve large SHG signals and increase SHG conversion efficiency, generating intense EF enhancement at energies of both $h v$ and $2 h v$ is highly desirable (i.e., photonic structures with resonant wavelengths at both $\lambda_{\mathrm{FW}}$ and $0.5 \lambda_{\mathrm{FW}}$ ). Based on our designs, by sequentially varying the values of $D$ and $P$, it is simple to obtain 

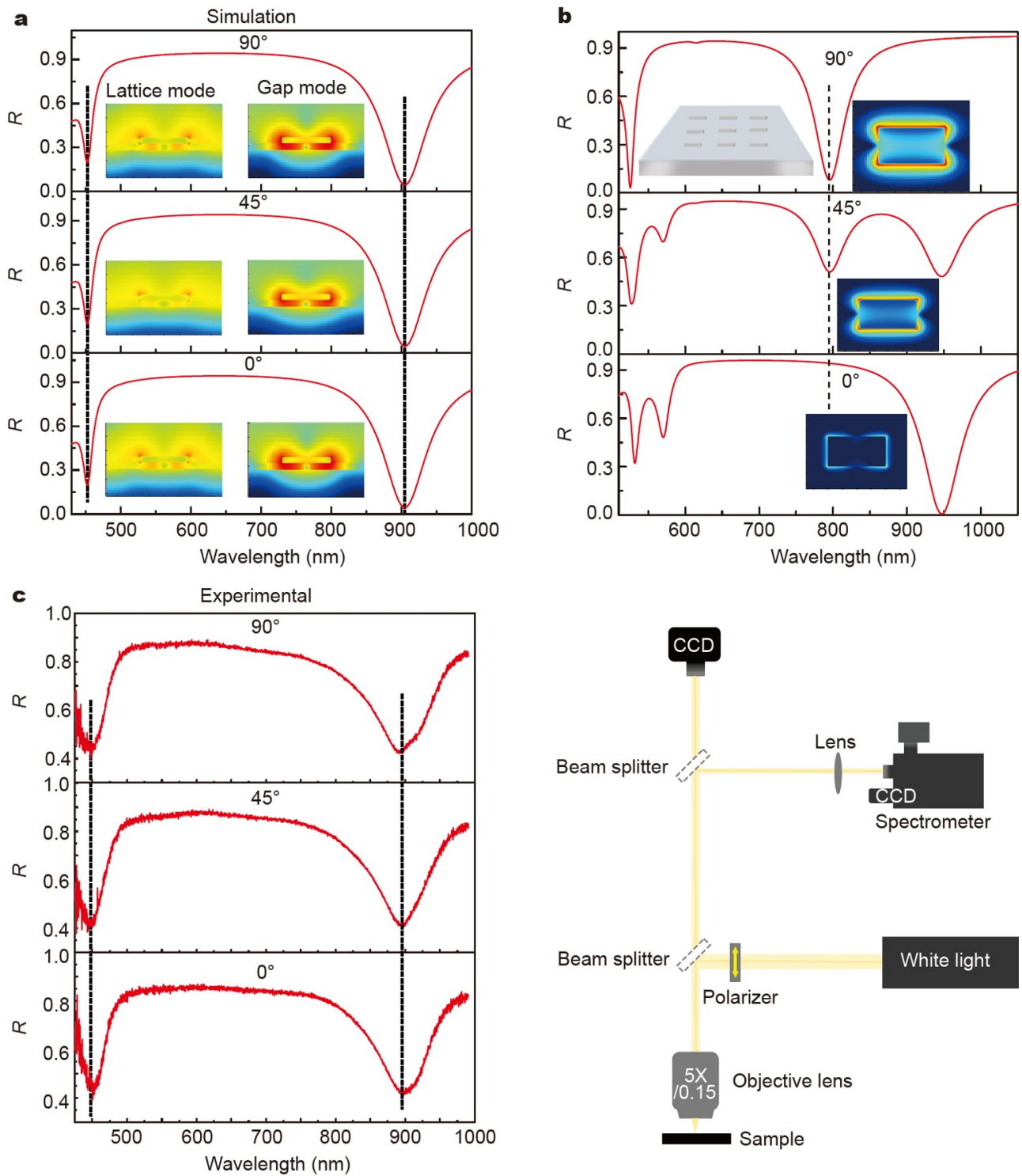

Figure 3 Characterizations of polarization independence. (a) Simulated reflection spectra of our abovementioned DMMs with the incidence of different polarization angles $\left(0^{\circ}, 45^{\circ}\right.$, and $\left.90^{\circ}\right)$. Inset: the $\mathrm{EF}$ distribution of the lattice and gap modes corresponding to polarization angles of $0^{\circ}$, $45^{\circ}$, and $90^{\circ}$ (the $x-z$ plane for $0^{\circ}$ and $45^{\circ}$ and the $y-z$ plane for $90^{\circ}$ ). The arrows in the insets represent the polarization direction. (b) The simulated reflection spectra of the nanorod array with an incident polarization varying between $0^{\circ}, 45^{\circ}$, and $90^{\circ}$. Insets on the right: the EF distributions around the nanorod at the upper interface of the gap-dielectric layer, with a wavelength marked by the dashed line. Top-left inset: schematic diagram of the nanorods array; the length of the nanorod is $200 \mathrm{~nm}$, the width is $140 \mathrm{~nm}$, the height is $20 \mathrm{~nm}$, and the period of the array is $370 \mathrm{~nm}$. (c) Experimental reflection spectra of our DMMs with incident polarization angles of $0^{\circ}, 45^{\circ}$, and $90^{\circ}$ (left), and the experimental setup schematic for the measurement (right).

on-demand gap and lattice modes of the DMMs at $\lambda_{\mathrm{FW}}$ and $0.5 \lambda_{\mathrm{FW}}$ (Fig. 4a), respectively.

The samples were fabricated via an EBL technique (more detailed information is presented in the EXPERIMENTAL SECTION), and Fig. 4b illustrates the measured reflection spectra of four typical samples. For each of the four samples, $\lambda_{\mathrm{L}}$ is half of $\lambda_{\mathrm{G}}$, which readily meets the requirements of SHG enhancement. Fig. $4 \mathrm{c}$ shows the
SEM images of typical DMMs with a nanodisk diameter of $145 \mathrm{~nm}$ and an array period of $350 \mathrm{~nm}$. It is apparent that the nanodisks are well organized in the tetragonal array. Because of the matched dual resonance and the strong field enhancement, SHG signals can be easily generated from the DMM (Fig. 4d). To characterize the enhancement effect of the DMMs, we applied reference samples with similar geometries (the inset in Fig. 4e), 

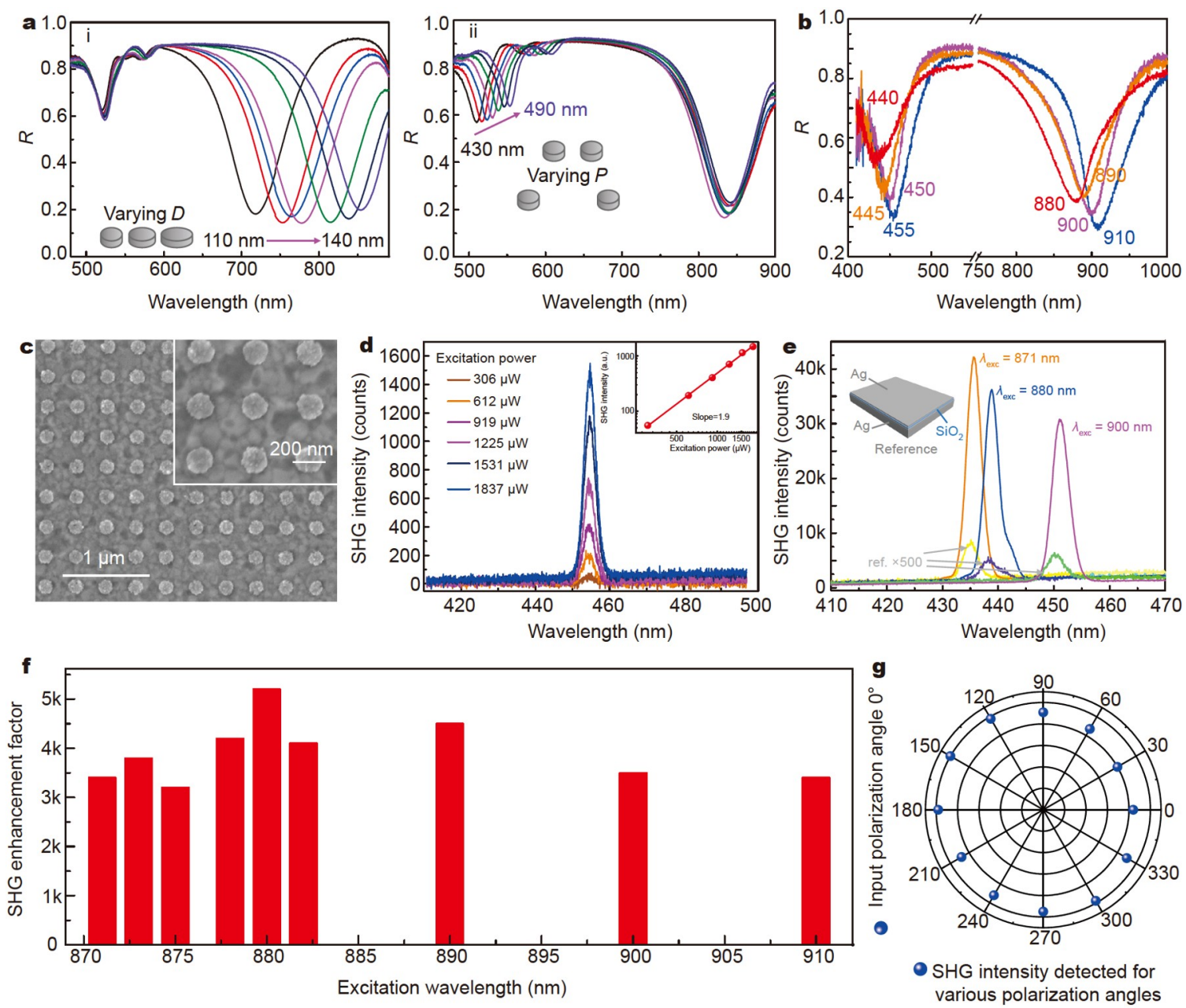

Figure 4 Employment of dual-mode metasurfaces for SHG enhancement. (a) Measured reflection spectra of the DMMs under variation of $D$ and $P$. Essentially, one can separately vary the values of $D$ and $P$, making $\lambda_{\mathrm{G}}$ and $\lambda_{\mathrm{L}}$ match $\lambda_{\mathrm{FW}}$ and $0.5 \lambda_{\mathrm{FW}}$, respectively. (i) The $\lambda_{\mathrm{G}}$ red shifts when $D$ changes from 110 to $140 \mathrm{~nm}$, with $P$ fixed at $450 \mathrm{~nm}$. (ii) The $\lambda_{\mathrm{L}}$ red shifts when $P$ changes from 430 to $490 \mathrm{~nm}$, with $D$ fixed at $140 \mathrm{~nm}$. (b) The measured reflection spectra of four typical DMMs for SHG enhancing, all of which meet the condition $\lambda_{\mathrm{L}}=0.5 \lambda_{\mathrm{G}}$. (c) SEM image of the DMMs fabricated using the EBL technique. Inset: upper right-hand corner is the top view. (d) SHG spectra of the DMMs with $\lambda_{\mathrm{L}}=455 \mathrm{~nm}$ and $\lambda_{\mathrm{G}}=910 \mathrm{~nm}$, under increasing excitation power. Inset: power dependence of the SHG peak intensity; the cure slope value of 1.9 indicates a second-order nonlinear optical process. (e) SHG spectra of DMMs and their reference sample. Inset: the scheme of the reference sample, which is a sandwich heterostructure of the Ag nanofilm $/ \mathrm{SiO}_{2} / \mathrm{Ag}$ film. (f) Measured SHG enhancement factor of the double-matching DMMs with different excitation wavelengths. (g) Measured SHG intensities for different detection polarizations, with an incident polarization angle of $0^{\circ}$.

which is also a sandwich heterostructure of the Ag nanofilm $/ \mathrm{SiO}_{2} / \mathrm{Ag}$ substrate, where the $\mathrm{Ag}$ nanofilm and $\mathrm{SiO}_{2}$ layer have the same thicknesses as the Ag nanodisk and dielectric layer in the DMM, respectively. In experiments, we separately collect the SHG intensities of the DMMs $\left(I_{\mathrm{DMM}}\right)$ and a reference sample $\left(I_{\mathrm{ref}}\right)$ and find that those from the DMMs are much stronger (Fig. 4e). To be more quantitative, we measured and calculated the SHG enhancement factors $\left(I_{\mathrm{DMM}} / I_{\mathrm{ref}}\right)$ of nine DMMs with $\lambda_{\mathrm{L}}=$ $2 \lambda_{\mathrm{G}}$ compared with their corresponding reference samples (Fig. 4f). The detailed information of the samples, including their structural parameters, reflection spectra, and SHG spectra, can be found in Figs S10 and S11. Generally, the SHG enhancement factors range from $4 \times$ $10^{3}$ to $5 \times 10^{3}$, and the largest SHG conversion efficiency is calculated as $6.9 \times 10^{-9} \mathrm{~W}^{-1}$ or $2.8 \times 10^{-6}$ (calculation details are illustrated in Note 1 of the Supplementary information). Such SHG conversion efficiency is distinctly higher than that of single-resonance structures (Fig. S12) and is almost 1-3 orders of magnitude higher than the previous records obtained in plasmonic systems (Table S2), thereby confirming the advantage of our 
DMMs for optical enhancements.

The large optical enhancements demonstrate the advantages of the dual-mode resonances, which not only support two resonant modes with strong EF enhancements but also enable these two modes to function together. Such advantages give rise to tremendous potential for the study of enhanced light-matter interactions. For example, apart from the optical enhancement in the SHG process demonstrated above, the DMMs can also be applied to enhancing spontaneous luminescence or other nonlinear processes, such as third-harmonic generation and multiphoton upconversion. Thus, although the SHG conversion efficiency of the DMMs is lower than those of emerging dielectric nonlinear metasurfaces $[39,40]$, the importance and applicability of DMMs for enhancing light-matter interactions cannot be doubted. Moreover, because of the special origin of SHG in metallic nanosystems (i.e., SHG induced by surface anisotropy [35,41]), we find that the second-harmonic emissions and excitations of our DMMs are polarization-independent.

More particularly, it is well known that SHG is a nonlinear optical process that depends upon second-order nonlinear susceptibility $\chi^{(2)}$; to avoid $\chi^{(2)}=0$, a lack of centrosymmetry is required for an SHG system. Therefore, for the majority of SHG systems, non-centrosymmetric media are applied, making the intensity of SHG polarization dependent. However, for plasmonic metals such as $\mathrm{Au}$ and $\mathrm{Ag}$, although metal atoms are actually organized in a face-centered cubic lattice with a centrosymmetric unit pattern, centrosymmetry breaking is locally induced at the metal surface because of the finite dimension of the atomic lattice. Thereafter, the plasmonic systems offer the rare possibility of an SHG that is insensitive to excitation polarization. As demonstrated in Fig. 4g and Fig. S13, because of the high-symmetry structure of the Ag nanodisk, the SHG intensity is almost unchanged under varied collecting polarizations. Moreover, the SHG intensity is stable under variation of the excitation polarization (Fig. S13), since the excitations and $\mathrm{EF}$ enhancements of the dual modes are unrelated to the excitation polarizations demonstrated in Fig. 3. This polarization independence property will benefit scenarios in which unpolarized light is preferred [42-44].

As demonstrated above, DMMs can act as a powerful platform for optical enhancement when the two resonant modes are located in two distinct areas, separately matching the pumping and emission wavelengths of an optical behavior. Conversely, if the two modes are designed to be spectrally overlapping, DMMs can also serve as ideal systems for studying modal strong coupling. It is well known that, when strong coupling appears between two resonant modes, a reversible exchange of energy between these modes will enable new hybrid modes with unique features [45-47]. Such modal strong coupling holds promising potential for quantum information processing and has been shown to be an efficient way to modulate the dephasing time, boost the Purcell effect, enhance water splitting, or improve energy transfer [4850]. To achieve such modal strong coupling, generating two kinds of resonant modes that overlap each other in both spectrum and space is essential. As for the DMMs, the realization of modal strong coupling becomes an easy task, thanks to the high spectral tunability of modes with a good spatial overlap of EF distributions. By tuning the gap mode such that it overlaps the lattice mode, a strong modal coupling is subsequently achieved, and the splitting energy can also be effectively modulated (Fig. S14). A detailed discussion can be found in Figs S14-S16, and N2 in the Supplementary information.

\section{CONCLUSIONS}

In summary, we have theoretically designed and experimentally demonstrated a new type of on-demand DMM by combining the surface lattice resonance with gap plasmons. These two modes can not only be independently tuned in remarkably wide spectral ranges but also significantly overlap spatially for EF enhancements. Additionally, with the involvement of structural symmetry, the DMMs showed polarization independence from the excitation light, which increases the generality for applications. With these advantages, our DMMs were able to work as a versatile platform for light-matter interactions, as exemplified by the achievement of a significant enhancement of SHG and the realization of the strong modal coupling. With excellent performances, the DMMs exemplified an innovative means of constructing a versatile platform for light-matter interactions; this may inspire new designs for functional multimodal photonic structures.

Received 20 January 2021; accepted 15 April 2021; published online 23 June 2021

1 Krasavin AV, Ginzburg P, Zayats AV. Free-electron optical nonlinearities in plasmonic nanostructures: A review of the hydrodynamic description. Laser Photonics Rev, 2018, 12: 1700082

2 Wu B, Wang PF, Qiu YH, et al. Enhanced second-harmonic generation of asymmetric Au@CdSe heterorods. Sci China Mater, 2020, 63: 1472-1479

3 Zhang X, Cao QT, Wang Z, et al. Symmetry-breaking-induced nonlinear optics at a microcavity surface. Nat Photon, 2019, 13: $21-24$ 
4 Bao Y, Yu Y, Xu H, et al. Full-colour nanoprint-hologram synchronous metasurface with arbitrary hue-saturation-brightness control. Light Sci Appl, 2019, 8: 95

5 Xue J, Zhou ZK, Lin L, et al. Perturbative countersurveillance metaoptics with compound nanosieves. Light Sci Appl, 2019, 8: 101

6 Panoiu NC, Sha WEI, Lei DY, et al. Nonlinear optics in plasmonic nanostructures. J Opt, 2018, 20: 083001

7 Li GC, Zhang Q, Maier SA, et al. Plasmonic particle-on-film nanocavities: a versatile platform for plasmon-enhanced spectroscopy and photochemistry. Nanophotonics, 2018, 7: 1865-1889

8 Liu SD, Leong ESP, Li GC, et al. Polarization-independent multiple Fano resonances in plasmonic nonamers for multimode-matching enhanced multiband second-harmonic generation. ACS Nano, 2016, 10: 1442-1453

9 Chikkaraddy R, de Nijs B, Benz F, et al. Single-molecule strong coupling at room temperature in plasmonic nanocavities. Nature, 2016, 535: 127-130

10 Karimpour S, Noori M. All-angle self-collimation-based invisibility cloaking in 2D square lattice photonic crystals. Annalen Der Physik, 2020, 532: 2000026

11 Tang Y, Liu Z, Deng J, et al. Nano-Kirigami metasurface with giant nonlinear optical circular dichroism. Laser Photonics Rev, 2020, 14: 2000085

12 Zhang $\mathrm{T}$, Che $\mathrm{Y}$, Chen $\mathrm{K}$, et al. Anapole mediated giant photothermal nonlinearity in nanostructured silicon. Nat Commun, 2020, 11: 3027

13 Bao Y, Jiang Q, Kang Y, et al. Enhanced optical performance of multifocal metalens with conic shapes. Light Sci Appl, 2017, 6: e17071

14 Uppu R, Eriksen HT, Thyrrestrup H, et al. On-chip deterministic operation of quantum dots in dual-mode waveguides for a plugand-play single-photon source. Nat Commun, 2020, 11: 3782

15 Peruzzo A, Laing A, Politi A, et al. Multimode quantum interference of photons in multiport integrated devices. Nat Commun, 2011, 2: 224

16 Fikouras AH, Schubert M, Karl M, et al. Non-obstructive intracellular nanolasers. Nat Commun, 2018, 9: 4817

17 Kravets VG, Schedin F, Jalil R, et al. Singular phase nano-optics in plasmonic metamaterials for label-free single-molecule detection. Nat Mater, 2013, 12: 304-309

18 Jaffe $\mathrm{T}$, Sorias $\mathrm{O}$, Gal L, et al. Doubly resonant nanoantennas on diamond for spatial addressing of spin states. Nano Lett, 2017, 17: 4217-4222

19 Weber N, Protte M, Walter F, et al. Double resonant plasmonic nanoantennas for efficient second harmonic generation in zinc oxide. Phys Rev B, 2017, 95: 205307

20 Liu SD, Yue P, Zhu MQ, et al. Restoring the silenced surface second-harmonic generation in split-ring resonators by magnetic and electric mode matching. Opt Express, 2019, 27: 26377-26391

21 Ciraci C, Hill RT, Mock JJ, et al. Probing the ultimate limits of plasmonic enhancement. Science, 2012, 337: 1072-1074

22 Li GC, Zhang YL, Lei DY. Hybrid plasmonic gap modes in metal film-coupled dimers and their physical origins revealed by polarization resolved dark field spectroscopy. Nanoscale, 2016, 8: 71197126

23 Li GC, Zhang YL, Jiang J, et al. Metal-substrate-mediated plasmon hybridization in a nanoparticle dimer for photoluminescence linewidth shrinking and intensity enhancement. ACS Nano, 2017, 11: 3067-3080
24 Zou S, Janel N, Schatz GC. Silver nanoparticle array structures that produce remarkably narrow plasmon lineshapes. J Chem Phys, 2004, 120: 10871-10875

25 Kravets VG, Schedin F, Grigorenko AN. Extremely narrow plasmon resonances based on diffraction coupling of localized plasmons in arrays of metallic nanoparticles. Phys Rev Lett, 2008, 101: 087403

26 Auguié B, Barnes WL. Collective resonances in gold nanoparticle arrays. Phys Rev Lett, 2008, 101: 143902

27 Farjadpour A, Roundy D, Rodriguez A, et al. Improving accuracy by subpixel smoothing in the finite-difference time domain. Opt Lett, 2006, 31: 2972-2974

28 Palik ED. Handbook of Optical Constants of Solids. San Diego: Academic Press, 1998

29 Celebrano $\mathrm{M}, \mathrm{Wu} \mathrm{X}$, Baselli $\mathrm{M}$, et al. Mode matching in multiresonant plasmonic nanoantennas for enhanced second harmonic generation. Nat Nanotech, 2015, 10: 412-417

30 Liu Z, Ye J. Highly controllable double Fano resonances in plasmonic metasurfaces. Nanoscale, 2016, 8: 17665-17674

31 Zeng Y, Qian H, Rozin MJ, et al. Enhanced second harmonic generation in double-resonance colloidal metasurfaces. Adv Funct Mater, 2018, 28: 1803019

32 Rodrigo D, Tittl A, John-Herpin A, et al. Self-similar multiresonant nanoantenna arrays for sensing from near- to midinfrared. ACS Photonics, 2018, 5: 4903-4911

33 Aouani H, Navarro-Cia M, Rahmani M, et al. Multiresonant broadband optical antennas as efficient tunable nanosources of second harmonic light. Nano Lett, 2012, 12: 4997-5002

34 Länk NO, Verre R, Johansson P, et al. Large-scale silicon nanophotonic metasurfaces with polarization independent near-perfect absorption. Nano Lett, 2017, 17: 3054-3060

35 Aydin K, Ferry VE, Briggs RM, et al. Broadband polarizationindependent resonant light absorption using ultrathin plasmonic super absorbers. Nat Commun, 2011, 2: 517

36 Butet J, Brevet PF, Martin OJF. Optical second harmonic generation in plasmonic nanostructures: From fundamental principles to advanced applications. ACS Nano, 2015, 9: 10545-10562

37 Ding SJ, Zhang H, Yang DJ, et al. Magnetic plasmon-enhanced second-harmonic generation on colloidal gold nanocups. Nano Lett, 2019, 19: 2005-2011

38 Zhang S, Li GC, Chen Y, et al. Pronounced Fano resonance in single gold split nanodisks with $15 \mathrm{~nm}$ split gaps for intensive second harmonic generation. ACS Nano, 2016, 10: 11105-11114

39 Liu S, Vabishchevich PP, Vaskin A, et al. An all-dielectric metasurface as a broadband optical frequency mixer. Nat Commun, 2018, 9: 2507

40 Koshelev K, Kruk S, Melik-Gaykazyan E, et al. Subwavelength dielectric resonators for nonlinear nanophotonics. Science, 2020, 367: 288-292

41 Wang Y, Wei D, Zhu Y, et al. Conversion of the optical orbital angular momentum in a plasmon-assisted second-harmonic generation. Appl Phys Lett, 2016, 109: 081105

42 Kim KH, Park BH, Tu Y, et al. Polarization-sensitive optical frequency domain imaging based on unpolarized light. Opt Express, 2011, 19: 552-561

43 Hisano K, Ota M, Aizawa M, et al. Single-step creation of polarization gratings by scanning wave photopolymerization with unpolarized light. J Opt Soc Am B, 2019, 36: D112-118

44 Lindfors K, Priimagi A, Setälä T, et al. Local polarization of tightly focused unpolarized light. Nat Photon, 2007, 1: 228-231 
Törmä P, Barnes WL. Strong coupling between surface plasmon polaritons and emitters: a review. Rep Prog Phys, 2015, 78: 013901

46 Christ A, Zentgraf T, Tikhodeev SG, et al. Controlling the interaction between localized and delocalized surface plasmon modes: Experiment and numerical calculations. Phys Rev B, 2006, 74: 155435

47 Farhang A, Bigler N, Martin OJF. Coupling of multiple LSP and SPP resonances: interactions between an elongated nanoparticle and a thin metallic film. Opt Lett, 2013, 38: 4758-4761

48 Yang J, Sun Q, Ueno K, et al. Manipulation of the dephasing time by strong coupling between localized and propagating surface plasmon modes. Nat Commun, 2018, 9: 4858

49 Shi X, Ueno K, Oshikiri T, et al. Enhanced water splitting under modal strong coupling conditions. Nat Nanotech, 2018, 13: 953958

50 Zhou ZK, Liu J, Bao Y, et al. Quantum plasmonics get applied. Prog Quantum Electron, 2019, 65: 1-20

Acknowledgements This work was supported by the National Key R\&D Program of China (2016YFA0301300), the National Natural Science Foundation of China (11974437 and 91750207), the Key-Area Research and Development Program of Guangdong Province (2018B030329001), Guangdong Special Support Program (2017TQ04C487), Guangdong Natural Science Funds for Distinguished Young Scholars (2017B030306007), Guangdong Natural Science Funds (2020A0505140004), Pearl River S\&T Nova Program of Guangzhou (201806010033), the Open Fund of IPOC (BUPT) (IPOC2019A003), and the Fundamental Research Funds for the Central Universities (20lgzd30).

Author contributions Zhou ZK conceived the idea of the research. Zhou ZK, Lin L and Xue J designed the research processes. Lin L and Xue J conducted most of the experiments and simulations. Xu $\mathrm{H}$ participated in the fabrication process. Zhao Q, Zhang $\mathrm{W}$ and Zheng $\mathrm{Y}$ performed the simulation of modal strong coupling. Zhou ZK, Wu L, Lin $\mathrm{L}$ and Xue $\mathrm{J}$ analyzed the results and wrote the manuscript. All authors contributed to the discussion of the manuscript.

Conflict of interest The authors declare no conflict of interest.

Supplementary information Supporting data are available in the online version of the paper.

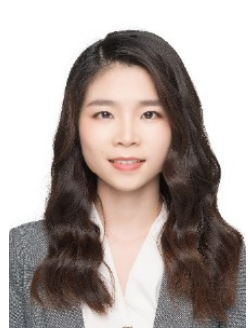

Limin Lin is currently a master candidate at Sun Yat-sen University. She received her B.Eng. (majored in information engineering) from the Department of Optoelectronic Engineering, Jinan University in 2018. Her master research focuses on optical metamaterials and their nonlinear device applications.

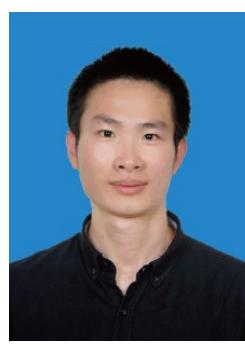

Jiancai Xue received his $\mathrm{PhD}$ degree (majored in optics) and BSc degree (majored in optical information science and technology) from Sun Yat-sen University in 2018 and 2013, respectively. He has been a postdoctoral fellow at Sun Yat-sen University since 2018. His research focuses on nanophotonics, including plasmonic devices, metasurfaces and functional nanostructures.

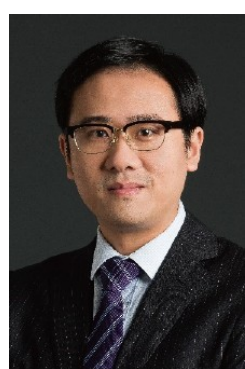

Zhang-Kai Zhou received his $\mathrm{PhD}$ degree in physics from Wuhan University in 2011 and joined Sun Yat-sen University in the same year. $\mathrm{He}$ is currently a professor of physics, as well as the deputy dean of the Department of Optics and Optical Engineering, School of Physics, Sun Yatsen University. His research interests mainly focus on the field of meta-optics, including quantum plasmonics, optical metamaterials, and functional nanodevices based on metasurfaces.

\section{基于等离激元晶格和间隙模式构建双共振超构表 面及其光与物质相互作用增强研究}

林丽敏 ${ }^{1 \dagger}$, 薛建材 ${ }^{1 \dagger}$, 徐昊飞 ${ }^{1}$, 赵骞 ${ }^{1}$, 张文博 ${ }^{1}$, 郑雅芹 ${ }^{1}$, 吴琳 ${ }^{2}$, 周张凯 ${ }^{1 *}$

摘要 克服光子结构中只有单个可控共振模式的问题对于增强光物相互作用和实现先进光子器件具有重要意义. 然而, 由于模式调 控的可调范围有限、电场增强的空间分布差异或模式激发具有偏 振依赖等因素的限制, 构建可调的多共振的光子结构一直受到限 制. 为了克服这些局限, 本文将等离激元表面晶格共振模式和间隙 等离激元模式相结合, 构建了一种双共振超表面体系, 该体系具有 覆盖可见光到近红外波段的光谱可调范围以及良好的电场增强空 间重叠性的特点. 为了证明该双共振超构表面的优势, 我们研究了 该体系的二次谐波效应，发现该双共振超构表面的二次谐波转化 效率比以往等离激元体系效率高 $1-3$ 个数量级. 以上结果显示, 我 们的发现为制备基于多模式共振光子结构的功能化器件提供了一 个有效方案. 